_ Der Versuch, die Politik und insbesondere Bundesgesundheitsminister Daniel Bahr auf die Seite der Ärzte zu ziehen, ist zunächst missglückt. Bahr äußerte lediglich sein Unverständnis über den Verlauf der „Honorarverhandlungen“. Die Grünen warnten sogar ausdrücklich davor, die hohen GKV-Überschüsse quasi als FDPWahlgeschenk an Ärzte und Apotheker zu verwenden.

_ Die KBV will den Kassen-Monopolverband weiter zwingen, sein Selbstverständnis zu offenbaren.

_ Klage der KBV vor dem Landessozialgericht Berlin/Brandenburg gegen den Erweiterten Bewertungsausschuss. Die Klage hat aufschiebende Wirkung. Hauptargument der KBV: Nach den gesetzlichen Bestimmungen hätte der Bewertungsausschuss Kostensteigerungen der Jahre 2008 bis 2012 berücksichtigen müssen. Die gesetzliche Vorschrift, dass 2011 und 2012 keine Anpassungen aus Kostengründen erfolgen dürfen, war zeitlich begrenzt - aber nun müssten Kostensteigerungen in vollem Umfang nachgeholt werden. Juristen halten die Klage für wohlbegründet.

_ Druck von außen - und da sind die freien Ärzteverbände im Spiel. Sie loten gegenwärtig aus, welche Protestmaßnahmen hin bis zu Praxisschließungen die Kassen zum Einlenken bewegen können. Das Spektrum tauglicher Aktionen dürfte sehr begrenzt sein: Getroffen werden müsste eigentlich der GKV-Spitzenverband, der aber weder mit der Versorgung noch mit Patienten und Versicherten direkt zu tun hat.

Ob sich die Kassen vom Ärzteprotest bewegen lassen, auf ihren Spitzenverband Druck auszuüben, ist höchst ungewiss. Auf dem Rücken der Patienten soll der Protest nach Möglichkeit auch nicht stattfinden. Hinzu kommt eines: Die Festlegung eines Orientierungswertes für die regionale Euro-Gebührenordnung ist nur ein Element der Weiterentwicklung von Honoraren: Auf KV-Ebene stehen seit diesem Jahr mit dem Versorgungsstrukturgesetz mehr Gestaltungsmöglichkeiten zur Verfügung. Insofern ist der Honorarpoker noch lange nicht $\mathrm{zu}$ Ende.

GKV behält Überschuss von mittlerweile 21,8 Milliarden $€$ weiter unter Verschluss

Der Überschuss der gesetzlichen Krankenkassen ist im ersten Halbjahr auf 2,7 Milliarden $€$ gestiegen. Die Finanzreserven, die sich bei den Einzelkassen türmen, erreichen damit 12,8 Milliarden $€$, weitere 9 Milliarden $€$ liegen im Gesundheitsfonds. Die Zahl der Kassen, deren Liquiditätspolster mehr als die zulässigen 1,5 Monatseinnahmen beträgt, hat sich nach Angaben des Bundesgesundheitsministeriums weiter erhöht. Nach mehrmaliger Aufforderung von Bundesgesundheitsminister Daniel Bahr (FDP) hat nun die Techniker Krankenkasse (TK) als die erste große Kasse - nach einer Reihe von Betriebskrankenkassen - grundsätzlich beschlossen, ihren Versicherten eine Prämie zurückzuerstatten. Die Höhe soll der Verwaltungsrat im Oktober bestimmen. Die TK hat im vergangenen Jahr einen Überschuss von 973 Millionen $€$ erzielt, ihre Reserven liegen bei fast 3 Milliarden $€$. Während Bahr glaubt, dass nun weitere Kassen dem Beispiel der TK folgen werden, lehnten die Barmer GEK und der AOK-Bundesverband dagegen in erster Reaktion Prämienzahlungen an ihre Versicherten ab.

Im ersten Halbjahr 2012 sind die Leistungsausgaben um 3,2\% je Versicherten gestiegen. Der Zuwachs der Ausgaben für ärztliche Behandlung war mit 2,1 \% unterdurchschnittlich. Diese Zahl beruht allerdings noch auf Prognosen und nicht auf tatsächlichen Abrechnungen. Unterdurchschnittlich blieb auch die Ausgabenentwicklung für Arzneimittel mit 3,1\%. Die Kosten liegen immer noch 560 Millionen unter denen des ersten Halbjahres 2010. Ursächlich ist der im August 2010 auf $16 \%$ erhöhte gesetzliche Rabatt. Anders als die Arzneimittelhersteller sieht das Bundesgesundheitsministerium keinen Grund, diesen Rabatt wieder zu reduzieren. Helmut Laschet

\title{
G-BA verhängt Zulassungsstopp für Spezialisten
}

Ein paar kleinere Arztgruppen erhalten seit Anfang September keine Zulassung mehr. So will es der Gemeinsame Bundesausschuss (G-BA) bis 1. Januar 2013, ab dann soll es eine neue Bedarfsplanungsrichtlinie geben.

E nen sofortigen Zulassungsstopp für einige kleinere Arztgruppen hat der Gemeinsame Bundesausschuss Anfang September in Berlin beschlossen. Die Sperre betrifft Jugendpsychiater, Nuklearmediziner, Strahlentherapeuten, Neurochirurgen, Humangenetiker, Laborärzte, Reha-Ärzte, Pathologen und Transfusionsmediziner. Das Moratorium gilt ab sofort bis zum 1. Januar 2013. Ab dann soll es eine neue Bedarfsplanungsrichtlinie geben, die der GBA derzeit vorbereitet.

Grund für den Zulassungsstopp: Der G-BA habe die Notbremse ziehen müssen, nachdem seine Absicht bekannt geworden sei, auch kleine Fachgruppen in die Planung einzubeziehen. Die KBV habe darüber informiert, dass es in den genannten Fachgruppen daraufhin zu einem überproportionalen Anstieg von Zulassungsanträgen gekommen sei. Es habe sich die Zahl der Zulassungsanträge von Strahlentherapeuten im ersten Halbjahr 2012 zum gleichen Vorjahreszeitraum von 75 auf 125 erhöht, so der Entwurf der Beschlussbegründung.

Mit dem Moratorium ziehen G-BA und KBV die Lehre aus den Erfahrungen mit dem "Seehofer-Bauch“ 1994: Als Folge einer Übergangsfrist vor Niederlassungssperren hatten damals rund 11.000 Ärzte in letzter Minute eine Kassenzulassung erhalten.

Gegen den Beschluss laufen betroffene Arztgruppen Sturm. Alle Pathologen arbeiteten sowohl ambulant als auch stationär. Eine Beplanung der Arztgruppe wäre katastrophal, sagte der Pathologe Professor Werner Schlake. Dem Protest schlossen sich umgehend auch Nuklearmediziner, Radiologen und Laborärzte an. Sie argumentieren, als auftragnehmende Ärzte gebe es für sie keine Möglichkeit der Leistungsausweitung auf Kosten anderer Arztgruppen.

sun/af 